\title{
In Vivo Target RNA Specificity of Trans-Splicing Phenomena by the Group I Intron
}

\section{Min-Sun Song and Seong-Wook Lee*}

Department of Molecular Biology, Institute of Nanosensor and Biotechnology, Dankook University, Yongin 448701, Korea

\begin{abstract}
The Tetrahymena group I intron has been shown to employ a trans-splicing reaction and has been modified to specifically target and replace human telomerase reverse transcriptase ( $h T E R T$ ) RNA with a suicide gene transcript, resulting in the induction of selective cytotoxicity in cancer cells that express the target RNA, in animal models as well as in cell cultures. In this study, we evaluated the target RNA specificity of trans-splicing phenomena by the group I intron in mice that were intraperitoneally inoculated with hTERT-expressing human cancer cells to validate the anti-cancer therapeutic applicability of the group I intron. To this end, an adenoviral vector that encoded for the hTERT-targeting group I intron was constructed and systemically injected into the animal. 5'-end RACE-PCR and sequencing analyses of the trans-spliced cDNA clones revealed that all of the analyzed products in the tumor tissue of the virus-infected mice resulted from reactions that were generated only with the targeted hTERT RNA. This study implies the in vivo target specificity of the transsplicing group I intron and hence suggests that RNA replacement via a trans-splicing reaction by the group I intron is a potent anti-cancer genetic approach.
\end{abstract}

Keywords: cancer gene therapy, group I intron, hTERT, RACE PCR, RNA replacement, trans-splicing

The Tetrahymena group I intron has been shown to perform replacement of a specific disease-causative or -associated RNA with a transgene transcript that exerts a therapeutic effect through a trans-splicing reaction, specifically in cells that express the target RNA (Drude et al., 2007). Therefore, the trans-splicing group I introns have been developed as gene therapeutic tools to repair mutant transcripts in genetic or malignant diseases or to

${ }^{*}$ Corresponding author: E-mail SWL0208@dankook.ac.kr Tel +82-31-8005-3195, Fax +82-31-8005-4058 Accepted 15 June 2008 reprogram transcripts that are involved in virus-infected diseases to induce new gene activity in the viral RNAexpressing cells (Lan et al., 1998; Phylactou et al., 1998; Rogers et al., 2002; Ryu et al., 2003; Kastanos et al., 2004; Shin et al., 2004).

Moreover, we recently have developed a specific trans-splicing group I intron that can target and replace human telomerase reverse transcriptase (hTERT) RNA to induce transgene activity specifically in cancer cells that express the RNA (Kwon et al., 2005). The group I intron has been demonstrated to specifically mark tumor cells that express hTERT and furthermore to specifically and effectively mediate regression of tumor cells not only in vitro but in mice that have been xenografted with human tumors (Hong et al., 2008; Jeong et al., 2008). These data indicate that the hTERT RNA-targeting trans-splicing group I intron can be a potent gene therapeutic agent against human cancer.

A major concern in applying the trans-splicing group I intron into clinical settings as a gene therapeutic agent for cancer is the in vivo specificity of cancer cell regression. For such a specificity, target RNA-independent and nonspecific transgene induction by the group I intron should be avoided. In other words, mis-spliced products should not be generated by the group I intron. In this study, in order to evaluate the therapeutic feasibility of the hTERT-specific group I intron, we assessed the target RNA specificity of the trans-splicing phenomenon by the intron in mice that have been intraperitoneally xenografted with human cancer cells.

\section{Construction of Adenoviral Vector for hTERT-specific Group I Intron}

The expression vector that encodes for the hTERT-specific trans-splicing group I intron was constructed as previously described (Kwon et al., 2005; Song et al., 2006). In brief, the Rib21AS group I intron, which recognizes uridine at position 21 (U21) of hTERT RNA, was generated to harbor an extended internal guide sequence, which includes an internal guide sequence (IGS, 5'-GGCAGG-3'), an extension of the P1 helix, an additional 6-nt-long P10 helix, and a 325-nt-long antisense sequence that is complementary to the downstream region ( +30 to +354 residues) of the targeted $\mathrm{U} 21$ of hTERT RNA, In addition, cDNA, as a $3^{\prime}$ exon that encodes for the lac $Z$ gene, was inserted downstream of the modified group I intron expression construct (Fig. 1A). 


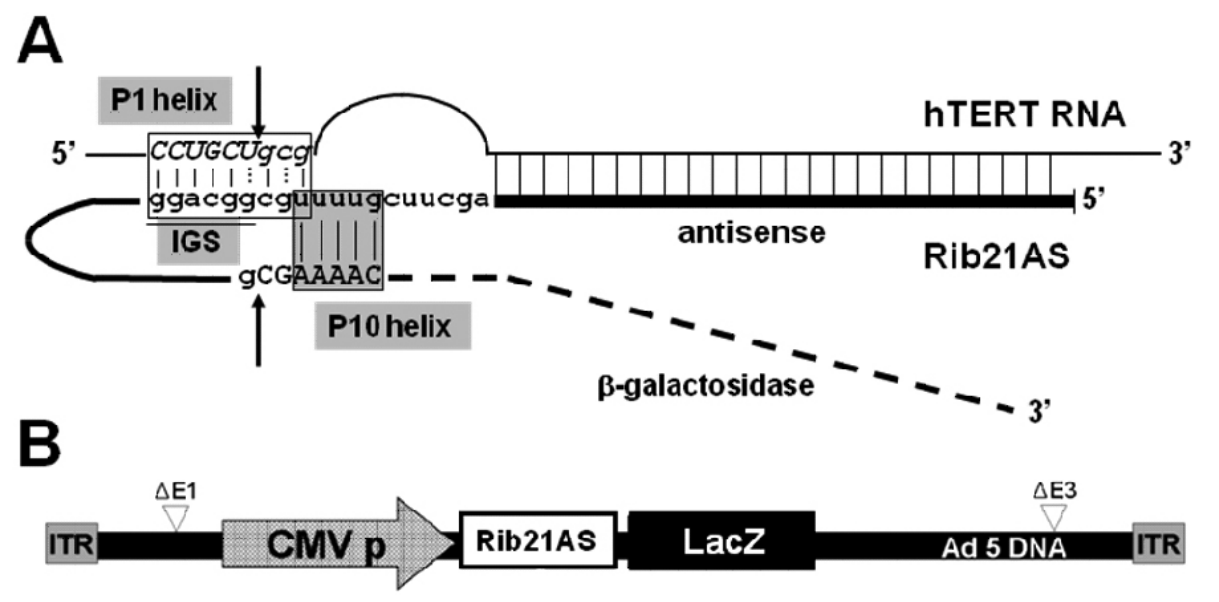

Fig. 1. Schematic diagram of the hTERT-specific trans-splicing group I intron and the adenoviral vector encoding for the intron. (A) The target hTERT RNA is presented with sequences around the splice site in italics. The group I intron, Rib21AS, is shown with extended IGS and $3^{\prime}$ exon sequences, $\beta$-galactosidase RNA, capitalized. Potential base parings between Rib21AS and the hTERT RNA are represented by vertical lines. Arrows indicate $5^{\prime}$ and $3^{\prime}$ splice sites. (B) Schematic diagram of recombinant adenoviral vector, Ad-CMV.Ribo-LacZ, encoding for the hTERT-specific Rib21AS under the control of the CMV promoter.

The Rib21AS group I intron has been observed to specifically and effectively induce transgene activity and, moreover, stimulate regression of $\mathrm{hTERT}^{+}$cancer cells not only in in vitro cultures but also in xenografted mice that have been transplanted with the cells (Kwon et al,, 2005; Hong et al., 2008; Jeong et al., 2008).

The resulting group I intron cDNA, flanked by the CMV promoter and the $3^{\prime}$ exon lac $Z$ sequence, was cloned into the $S p e l / B s t B I$ site of the pAdenoVator-CMV5IRES-GFP shuttle vector (Qbiogene, Irvine, CA). The recombinant adenoviral vector that encodes for the modified intron was then constructed via an in vivo homologous recombination method in bacteria (BJ5183) as follows. First, the modified shuttle vector was linearized with Pmel and then cotransformed into BJ5183 cells with an E1/E3-deleted type 5 adenoviral backbone genome (pAdenoVator $\triangle \mathrm{E} 1 / \mathrm{E} 3$, Qbiogene). The recombinant vector that was created through homologous recombination in the BJ5183 cells was isolated and linearized with Pad and then transfected into 293 cells. The produced recombinant adenovirus (Ad-CMV.Ribo-LacZ, Fig. 1B) was isolated via plaque purification, amplified using 293 cells, purified, and concentrated using Vivapure $^{\circledR}$ AdenoPACK ${ }^{\mathrm{TM}} 100$ (Sartorius AG, Edgewood, $N Y$ ). Titers of the recombinant adenovirus were determined by TCID50 analysis. The construct of the recombinant adenoviral vector was confirmed by PCR analysis of viral genomic DNA in hTERT $^{+} \mathrm{HT}^{-29}$ cells that were infected with Ad-CMV.Ribo-LacZ (data not shown). HTERT-specific expression of the transgene by the adenoviral vector was proven using the $\beta$-galactosi- dase assay on cell extracts and RT-PCR analysis of total RNA in the adenovirus-infected cells (data not shown).

\section{In Vivo Specificity of the Trans-splicing Reaction by the Group I Intron}

To assess the in vivo target RNA specificity of the hTERT-specific trans-splicing group I intron, we first established an animal model of peritoneal carcinomatosis by intraperitoneal inoculation of HT-29 cells into male BALB/c nude mice. The animals usually showed peritoneal carcinomatosis within 21 days after cell injection. We then infected $1 \times 10^{9}$ pfu of Ad-CMV Ribo-LacZ into the mice intraperitoneally, extracted total RNA from the tumors that were established in the mouse 2 days after the virus treatment, and analyzed the in vivo specificity of the group I intron at the molecular level by employing an RNA-tagging approach. In other words, we determined whether the intron was reacting with RNAs other than the targeted hTERT RNAs inside adenovirusinfected tumors. To address the question, we performed RT and a 5'-end RACE (rapid amplification of CDNA ends) PCR reaction of trans-spliced products in the hTERT-expressing tumors that were established in the animal (Fig. 2). Total RNA from the adenovirus-infected tumor tissues was reverse-transcribed with random primers. The $3^{\prime}$-ends of the cDNA products were then ligated with adaptor oligonucleotides (Takara, Otsu, Shiga, Japan). The modified cDNAs were amplified with 10 pmol adaptor as the $5^{\prime}$ primer (Takara) and a lac $Z$ 


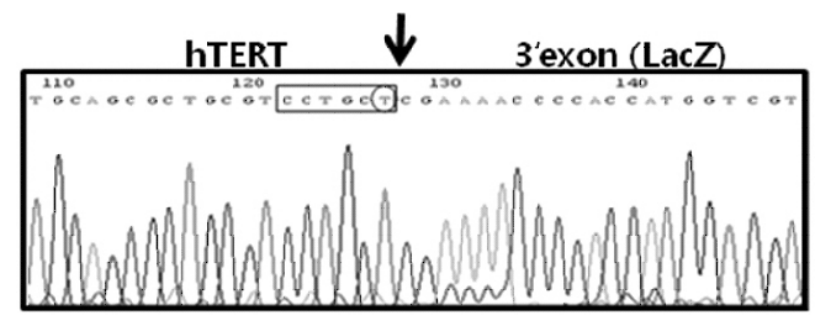

Fig. 2. Specificity of the in vivo trans-splicing reaction by a specific trans-splicing group I intron. A representative sequence of amplified products was shown from trans-spliced RNA in hTERT $^{+}$tumors of Ad-CMV.Ribo-LacZ infected mice. All of the amplified clones showed the same sequence pattern. The expected sequence, consisting of the splicing junction site, which is indicated with an arrow, is presented with the intron recognition sequence in the target RNA (boxed) and uridine at position 21 (circled).

3' primer (5'-GGGAAGCTTCGGATTGACCGTAATGGGA). The amplified cDNAs were then cloned, and 20 different clones were sequenced. Of note, the sequence of the analyzed splice products showed that all of the products resulted from trans-splicing reactions only with the targeted hTERT RNA, not with any other RNAs (Fig. 2). In sharp contrast, no trans-spliced products were produced in any of the normal tissues in the virus-infected mouse (data not shown). These results strongly imply that the group I intron specifically reacted in vivo with only the targeted cellular transcript, hTERT RNA, and exerted highly targeted RNA-specific trans-splicing activity; thus, no mis-spliced products were produced in the intron-delivered mice. Therefore, by using a specific trans-splicing group I intron, the possibility of nonspecific transgene induction in $\mathrm{hTERT}^{-}$normal cells, which might happen via off-target effects, can be minimized.

Importantly, the trans-splicing group I intron has more effective anti-cancer features because it can reduce cancer-related target RNA levels and simultaneously induce target-specific therapeutic gene expression. Together with its efficacy, the in vivo target specificity of the group I intron, which was confirmed at the molecular level in this study, suggests that cancer-specific RNA-targeted gene delivery approaches that are based on RNA replacement will be very useful and potent strategies to control human cancers.

\section{Acknowledgements}

The present research was conducted with the research fund of Dankook University in 2006.

\section{References}

Drude, I., Dombos, V., Vauleon, S., and Muller, S. (2007). Drugs made of RNA: development and application of engineered RNAs for gene therapy. Mini Rev. Med. Chem. 7, 912-931.

Hong, S.H., Jeong, J.S., Lee, Y.J., Jung, H.I., Cho, K.S., Kim, C.M., Kwon, B.S., Sullenger, B.A., Lee, S.W., and Kim, I.H. (2008). In Vivo reprogramming of hTERT by trans-splicing ribozyme to target tumor cells. Mol. Ther. $16,74-80$.

Jeong, J.S., Lee, S.W., Hong, S.H., Lee, Y.J., Jung, H.I., Cho, K.S., Seo, H.H., Lee, S.J., Park, S., Song, M.S., Kim, C.M., and Kim, I.H. (2008). Antitumor effects of systemically delivered adenovirus harboring trans-splicing ribozyme in intrahepatic colon cancer mouse model. Clin. Cancer Res. 14, 281-290.

Kastanos, E., Hjiantoniou, E., and Phylactou, L.A. (2004). Restoration of protein synthesis in pancreatic cancer cells by trans-splicing ribozymes. Biochem. Biophys, Res. Commun. 322, 930-934.

Kwon, B.S., Jung, H.S., Song, M.S., Cho, K.S., Kim, S.C., Kimm, K., Jeong, J.S., Kim, I.H., and Lee, S.W. (2005) Specific regression of human cancer cells by ribozymemediated targeted replacement of tumor-specific transcript. Mol. Ther. 12, 824-834.

Lan, N., Howrey, R.P., Lee, S.W., Smith, C.A., and Sullenger, B.A. (1998). Ribozyme-mediated repair of sickle $\beta$ globin mRNAs in erythrocyte precursors. Science 280, 1593-1596.

Phylactou, L.A., Darrah, C., and Wood, M.A.J. (1998). Ribozyme-mediated trans-splicing of a trinucleotide repeat. Nat. Genet. 18, 378-381.

Rogers, C.S., Vanoye, C.G., Sullenger, B.A., and George, A.L. (2002). Functional repair of a mutant chloride channel using a trans-splicing ribozyme. J. Clin. Invest. 110, 1783-1798.

Ryu, K.J., Kim, J.H., and Lee, S.W. (2003). Ribozyme-mediated selective induction of new gene activity in hepatitis $C$ virus internal ribosome entry site-expressing cells by targeted trans-splicing. Mol. Ther. 7, 386-395.

Shin, K.S., Sullenger, B.A., and Lee, S.W. (2004). Ribozyme-mediated induction of apoptosis in human cancer cells by targeted repair of mutant p53 RNA. Mol. Ther. 10, 365-372.

Song, M.S., and Lee, S.W. (2006). Cancer-selective induction of cytotoxicity by tissue-specific expression of targeted trans-splicing ribozyme. FEBS. Lett. 580, 50335043. 